\title{
Pollen Classification Based on Geometrical, Descriptors and Colour Features Using Decorrelation Stretching Method
}

\author{
Jaime R. Ticay-Rivas ${ }^{1}$, Marcos del Pozo-Baños ${ }^{1}$, Carlos M. Travieso ${ }^{1}$, \\ Jorge Arroyo-Hernández ${ }^{2}$, Santiago T. Pérez ${ }^{1}$, Jesús B. Alonso ${ }^{1}$, \\ and Federico Mora-Mora ${ }^{2}$ \\ ${ }^{1}$ Signals and Communications Department, \\ Institute for Technological Development and Innovation in Communications, \\ University of Las Palmas de Gran Canaria, \\ Campus University of Tafira, 35017, Las Palmas de Gran Canaria, Las Palmas, Spain \\ \{jrticay, mpozo, ctravieso, sperez, jalonso\}@idetic.eu \\ ${ }^{2}$ Escuela de Matemáticas, Universidad Nacional, Costa Rica \\ \{fmoram, jarroy\}@una.ac.cr
}

\begin{abstract}
Saving earth's biodiversity for future generations is an important global task, where automatic recognition of pollen species by means of computer vision represents a highly prioritized issue. This work focuses on analysis and classification stages. A combination of geometrical measures, Fourier descriptors of morphological details using Discrete Cosine Transform (DCT) in order to select their most significant values, and colour information over decorrelated stretched images are proposed as pollen grains discriminative features. A Multi-Layer neural network was used as classifier applying scores fusion techniques. 17 tropical honey plant species have been classified achieving a mean of $96.49 \% \pm 1.16$ of success.
\end{abstract}

Keywords: Pollen grains, pollen classification, colour features, geometrical features, neural networks, decorrelation stretch.

\section{Introduction}

Over $20 \%$ of all the world's plants are already at the edge of becoming extinct [1]. Saving earth's biodiversity for future generations is an important global task [2] and many methods must be combined to achieve this goal. Saving flora biodiversity involves mapping plant distribution by collecting pollen and identifying them in a laboratory environment. Pollen grain classification is a qualitative process, involving observation and discrimination of features.

The manual method, heavily depending on experts, is still the most accurate and effective, but takes considerable amounts of time and resources, limiting research progress [3]. Therefore, automatic recognition of pollen species by means of computer vision is a highly prioritized subject in palynology.

A lot of progress has been made in the field of automatic pollen species classification. In [4], it showed one of the first works in which texture features and 
neural networks were used for pollen grains identification task. In [5] detection and classification of pollen grains were identified as the main and difficult tasks. In both cases neural networks were used. The characteristics of pollen grains for their correct classification were studied in different studies. In [6], the shape and ornamentation of the grains were analyzed, using simple geometric measures. Concurrence matrices were applied for the measurement of texture in [7]. Both techniques were combined in [8] and [9]. In [10] brightness and shape descriptors were used as vector features.

Another interesting approach is found in [11]. Here, a combination of statistical reasoning, feature learning and expertise knowledge from the application was used. Feature extraction was applied alternating 2D and 3D representations. Iterative refinement of hypotheses was used during the classification process. A full 3D volume database of pollen grain was recorded in [12] using a confocal laser scanning microscope. 14 invariant gray-scale features based on integration over the 3D Euclidian transformation were extracted and used for classification. A more recent work with an ambitious approach is shown in [13]. It describes an automatic optical recognition and classification of pollen grains system. This is able to locate pollen grains on slides, focus and photograph them, and finally identify the species applying a trained neural network.

There is no doubt that significant progress has been made, but more knowledge is needed in the combination of features that have been studied and colour features in order to increase the number of species that systems are capable to recognize and classify with a high rate of success.

This work introduces the use of colour features over decorrelated images. A novel combination of these features with geometrical measures, and Fourier descriptors of morphological details are proposed as pollen grains discriminative features, being this our first work in this area. The remainder of this paper is organized as follows. In Section 2 the process of pollen grains detection is explained. Section 3 introduces the techniques used for feature extraction. The classification block is presented in Section 4. Experimental methodology and its results are exposed in Section 5. Finally in Section 6, discussions and conclusions are shown.

\section{Pollen Grains Detection}

Since there is not a uniform focus and conglomerates may appear in the images, a simple semi-automatic algorithm was developed to capture the pollen grains, indicating and cropping the pollen image. Thus, only one grain appears in each pollen grain sub-image. An example of each pollen class and its Scientist name (family, gender and specie by column) can be seen in figure 1 .

Once sub-images of the pollen grains have been selected, automatic detection is performed. First, the colour contrast of the image is increased in order to locate the pollen grain in the image. Then a decorrelation stretching (DS) of the image is done. This technique is explained in detail in [15] and [16]. The main idea is to achieve a variance-equalizing enhancement in order to uncorrelate and to fill the colour space.

The pollen grain image is represented by a matrix with dimensions $M \times N \times 3$ where $M \times N$ represents the image dimensions of each colour channel. 


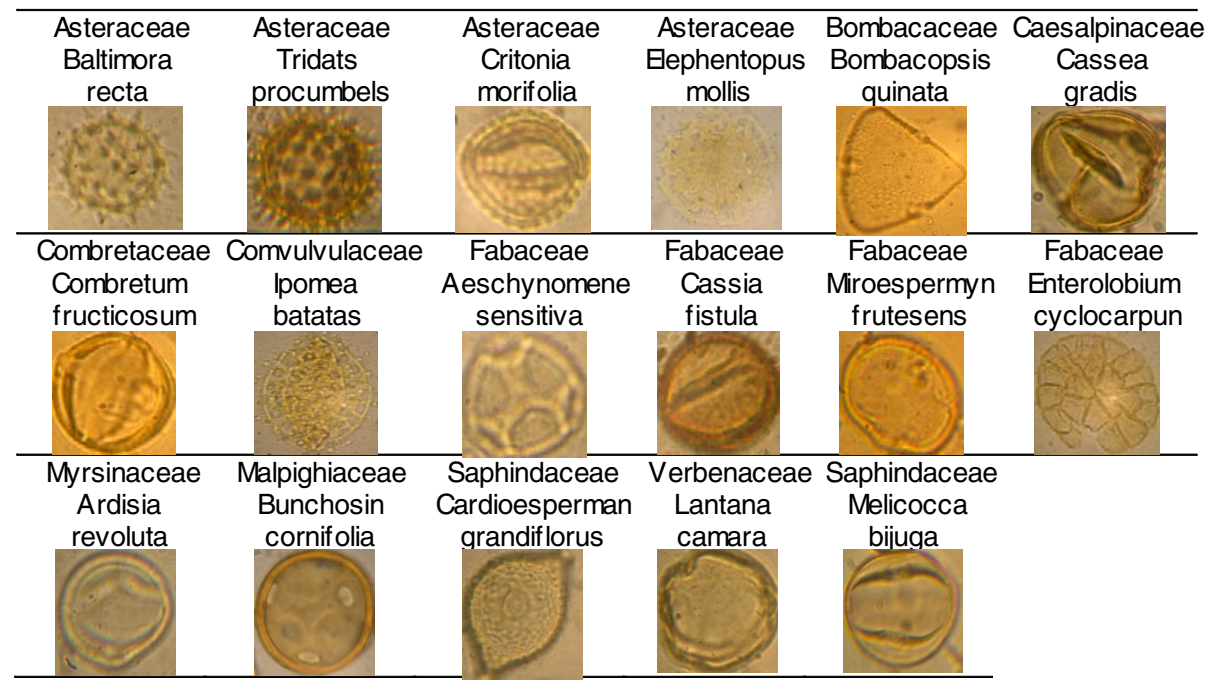

Fig. 1. Examples of each pollen class

Thus, the pixels of the image are represented by the vector $x \in \mathfrak{R}^{I}$. Let $\boldsymbol{V}$ express the covariance or correlation matrix of $x$ and the eigenanalysis associated [16] with the principal component analysis described as:

$$
V=U E U^{t}
$$

Where the columns of $U$ denote the eigenvectors and the diagonal elements of the diagonal matrix $E$ denote the corresponding eigenvalues. Using the transform $U^{t}$ the vector $x$ is transformed in a new vector space where the new data $y$ is given by:

$$
y=U^{t} x
$$

The scaling, applied in order to equalize the variance, is achieved in this space dividing each value of $y$ by its corresponding standard deviation $e_{i}^{1 / 2}$, thus the new $w$ matrix is

$$
w=E^{-1 / 2} U^{t} x
$$

where

$$
E=\operatorname{diag}\left(e_{1}, e_{2}, e_{3}\right)
$$

The transformation to the original space is given by

$$
z=U E^{-1 / 2} U^{t} x
$$


The resulting image is transformed to the HSV colour model (Hue, Saturation and Value). The $\mathrm{S}$ channel is used in order to take advantage of the difference of brightness between the background and the pollen grain. Histogram equalized image is necessary before the binarization of the image. The next factor is multiplied to the histogram equalization:

$$
f=1 / e^{((m+0.1) * 1.5)}+0.8
$$

where $m$ is the mean of the $\mathrm{S}$ channel insuring that the equalization is made in a correct range.

The binarization is applied setting the threshold at 0.45 . Morphological methods like close, erosion, dilation, etc. are used in order to consider only the area where the pollen grain is found. Figure 2 shows the obtained images:

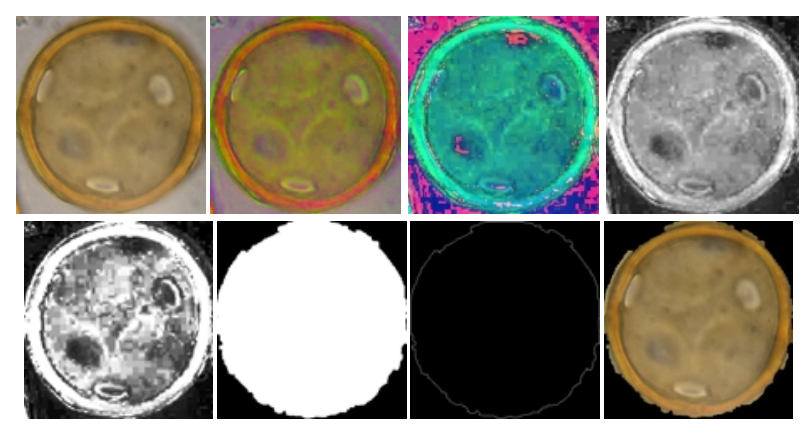

Fig. 2. Images obtained in the pollen grains detection

\section{Discriminative Feature Extractions}

Discriminative feature extractions were divided in three steps and are described in the next sections:

\subsection{Geometrical Features}

The geometrical features have been used in almost all works related to grain classification. The area, convex area, and perimeter are used in this work as they have been proven to provide a fair amount of the basic information of the pollen grain. Area is the number of pixels in the considered region. Convex area is the number of pixels of the binary image that specifies the convex hull. Perimeter is calculated as the distance between each adjoining pair of pixels around the border of the pollen grain. 


\subsection{Fourier Descriptors}

The Fourier Descriptor represents the shape of the pollen grain in the frequency domain [10], [16]. Each boundary point is represented as a complex number. In order to eliminate the bias effect, the centroid of the shape of the pollen grain is introduced in the complex representation:

$$
s=\left[x-x_{c}\right]+i\left[y-y_{c}\right]
$$

The contour is sampled each 2 degrees using the Cartesian to radian coordinates transformation. The Fourier Descriptor is defined as the power spectrum of the discrete Fourier Transform of $s$. In this work the Discrete Cosine Transformation (DCT) is applied to the resulting power spectrum in order to select the most significant values of the Fourier Descriptor. Finally, the first three coefficients of the transformation were used as features on this approach.

\subsection{Colour Features}

In section 2, decorrelation stretching method (DS) is applied to the pollen grain images. The averages of R (Red), B (Blue), $\mathrm{H}$ (Hue) channels were calculated as three colour parameters. It is important to mention that only the pollen grain region was considered. The Gray Level Co-occurrence Matrix (GLCM) from H channel was used in order to obtain its contrast. The distance and direction setting in the GLCM calculation was 1 pixel and 0 degree respectively.

\section{Classification System}

Artificial neural networks (NN) have been used in several studies [4], [5], [7], [8], [13] demonstrating good results. Multi-Layer Perceptron NN trained by Back Propagation algorithm (MLP-BP) were used. Neurons implemented the hyperbolic tangent sigmoid transfer function. The number of neurons in the input layer is the same as the number of selected features. Following experimentation, it was determined to use one hidden layer. The number of neurons that showed better results ranged between 20 and 80 . The best results were found using 50 neurons. The numbers of neurons in the output layer was 17, that is, the number of classes. 5000 iterations were performed in the training phase.

Since weights of neurons in the input layer were initialized randomly, $30 \mathrm{NNs}$ were trained in order to determine the classes. Two decision techniques (DT) were applied: class majority voting (CMV) and adding scores (AS). The former selects the most repeated result along all 30 NNs. These results at every NN are computed as the index of the maximum scoring neuron. The AS adds the output scores of corresponding neurons from each $30 \mathrm{NNs}$, before selecting the index of the maximum value. 


\section{Experiments and Results}

\subsection{Database}

The database contains a total of 426 pollen grain sub-images as those presented in figure 1. These sub-images correspond to 17 genders and species from 11 different families of tropical honey plants from Costa Rica, Central America.

\subsection{Experimental Methodology and Results}

Parameters introduced in section 3 were tested for both raw images and DS processed images. For each of them, apart from the original dataset of parameters (ORIG), a second dataset of parameters (ORIGPCA) was obtained by applying PCA to the original dataset. About the classification, CMV and AS decision techniques were applied separately. Moreover, both parameter sets were fused at the score level using again the AS technique. This makes a total of 8 combinations of raw/DS-processed images, original/PCA data set, and CVM/AS decision technique.

In order to obtain valid results, a series of cross-validation methods were performed on every experiment: 30 iterations of $50 \%$ hold out $(50-\mathrm{HO})$, and k-folds with k equal 3, 4, and 5 (3-, 4-, and 5-folds). A second set of features were obtained applying the DS method to the original images. Tables 1 and 2 show all obtained results for both original and DS processed set of images, showing results as mean $(\%)$ and standard desviation $( \pm)$ from 30 iterations.

Table 1. Results obtained for the original set of parameters and after applying PCA, for both CMV and AS score fusion methods

\begin{tabular}{lllllll}
\hline DS & PCA DT & 50-HO & 3-folds & 4-folds & 5-folds \\
\hline No & No & CMV & $86.25 \% \pm 2.67$ & $87.98 \% \pm 3.13$ & $90.01 \% \pm 3.77$ & $90.44 \% \pm 3.53$ \\
No & Yes & CMV & $86.13 \% \pm 3.00$ & $88.98 \% \pm 7.54$ & $91.79 \% \pm 7.01$ & $92.62 \% \pm 7.17$ \\
No & No & AS & $86.48 \% \pm 2.75$ & $88.87 \% \pm 2.70$ & $90.30 \% \pm 3.45$ & $90.19 \% \pm 3.84$ \\
No & Yes & AS & $86.55 \% \pm 3.08$ & $90.25 \% \pm 3.36$ & $93.34 \% \pm 4.74$ & $93.80 \% \pm 4.25$ \\
Yes & No & CMV & $88.08 \% \pm 1.73$ & $90.91 \% \pm 1.11$ & $90.98 \% \pm 2.45$ & $91.37 \% \pm 3.34$ \\
Yes & Yes & CMV & $93.99 \% \pm 1.63$ & $95.22 \% \pm 0.24$ & $94.22 \% \pm 2.76$ & $95.73 \% \pm 1.84$ \\
Yes & No & AS & $88.20 \% \pm 2.19$ & $90.91 \% \pm 0.62$ & $90.86 \% \pm 2.12$ & $91.76 \% \pm 3.27$ \\
Yes & Yes & AS & $94.18 \% \pm 1.71$ & $95.55 \% \pm 0.15$ & $95.36 \% \pm 1.88$ & $95.73 \% \pm 1.84$ \\
\hline
\end{tabular}

Table 2. Results obtained merging ORIG and ORIGPCA parameters using AS score fusion

\begin{tabular}{lllllll}
\hline Param. & DS & DT & $\mathbf{5 0 - H O}$ & 3-folds & 4-folds & 5-folds \\
\hline ORIG + No & AS & $91.19 \% \pm 2.07$ & $92.96 \% \pm 1.74$ & $94.61 \% \pm 3.05$ & $95.78 \% \pm 4.45$ \\
ORIGPCA & & & & & \\
ORIG + Yes & AS & $95.33 \% \pm 1.31$ & $96.53 \% \pm 0.21$ & $96.63 \% \pm 1.17$ & $97.48 \% \pm 1.97$ \\
ORIGPCA & & & & & \\
\hline
\end{tabular}




\section{Discussion and Conclusions}

This work has introduced the use of colour features over decorrelated stretched images, for enhance pollen grain classification. More standard characteristics like geometrical features and Fourier descriptors have been added to the pollen grain descriptions. Over this multiple feature vector, PCA has proven to increase the system's performance.

Experimental results shown in Table 1 indicate how the use of DS technique significantly improves the recognition of pollen grain species. Moreover, this improvement can be seen in terms of success rate and stability (standard deviation). This can be explained by the fact that colours channel variances are equalized and uncorrelated, reducing intra-class variation and differentiating inter-class samples. Furthermore, the use of PCA on the original database increased the success rate. This is consistent with the fact that PCA transformation projects samples over more meaningful dimensions, maximizing variance.

Finally, both data sets (ORIG and ORIGPCA) were fused using the AS score fusion, obtaining a maximum success rate of $96.4 \%$, and a standard deviation of 1.16 with 17 pollen species. It is worth to mention that these results improve those achieved by other authors [7] [8] [9] [10], even though the number of classified species was significantly larger.

Acknowledgements. This work has been supported by Spanish Government, in particular by "Agencia Española de Cooperación Internacional para el Desarrollo" under funds from D/027406/09 for 2010, and D/033858/10 for 2011.

To M.Sc. Luis A. Sánchez Chaves, Tropical Bee Research Center (CINAT) at Universidad Nacional de Costa Rica, for provides the database images and palynology knowledge.

\section{References}

1. Plants under pressure: a global assessment. The first report of the IUCN Sampled Red List Index for Plants. Royal Botanic Gardens, Kew, UK (2010)

2. Sytnik, K.M.: Preservation of biological diversity: Top-priority tasks of society and state. Ukrainian Journal of Physical Optics 11(suppl. 1), S2-S10 (2010)

3. Stillman, E.C., Flenley, J.R.: The Needs and Prospects for Automation in Palynology. Quaternary Science Reviews 15, 1-5 (1996)

4. Li, P., Flenley, J.: Pollen texture identification using neural networks. Grana 38(1), 59-64 (1999)

5. France, I., Duller, A., Duller, G., Lamb, H.: A new approach to automated pollen analysis. Quaternary Science Reviews 18, 536-537 (2000)

6. Treloar, W.J., Taylor, G.E., Flenley, J.R.: Towards Automation of Palynology 1: Analysis of Pollen Shape and Ornamentation using Simple Geometric Measures, Derived from Scanning Electron Microscope Images. Journal of Quaternary Science 19(8), 745-754 (2004)

7. Li, P., Treloar, W.J., Flenley, J.R., Empson, L.: Towards Automation of Palynology 2: The Use of Texture Measures and Neural Network Analysis for Automated Identification of Optical Images of Pollen Grains. Journal of Quaternary Science 19(8), 755-762 (2004) 
8. Zhang, Y., Fountain, D.W., Hodgson, R.M., Flenley, J.R., Gunetileke, S.: Towards Automation of Palynology 3: Pollen Pattern Recognition using Gabor Transforms and Digital Moments. Journal of Quaternary Science 19(8), 763-768 (2004)

9. Rodriguez-Damian, M., Cernadas, E., Formella, A., Fernandez-Delgado, M., De Sa-Otero, P.: Automatic detection and classification of grains of pollen based on shape and texture. IEEE Trans. on Systems, Man, and Cybernetics, Part C: Applications and Reviews 36(4), 531-542 (2006)

10. Rodriguez-Damian, M., Cernadas, E., Formella, A., Sa-Otero, R.: Pollen classification using brightness-based and shape-based descriptors. In: Proceedings of the 17th International Conference on Pattern Recognition, ICPR 2004, August 23-26, vol. 2, pp. 23-26 (2004)

11. Boucher, A., Thonnat, M.: Object recognition from 3D blurred images. In: 16th International Conference on Pattern Recognition, vol. 1, pp. 800-803 (2002)

12. Ronneberger, O., Burkhardt, H., Schultz, E.: General-purpose object recognition in 3D volume data sets using gray-scale invariants - classification of airborne pollen-grains recorded with a confocal laser scanning microscope. In: 16th International Conference on Pattern Recognition, vol. 2, pp. 290-295 (2002)

13. Allen, G.P., Hodgson, R.M., Marsland, S.R., Flenley, J.R.: Machine vision for automated optical recognition and classification of pollen grains or other singulated microscopic objects. In: 15th International Conference on Mechatronics and Machine Vision in Practice, December 2-4, pp. 221-226 (2008)

14. Karvelis, P.S., Fotiadis, D.I.: A region based decorrelation stretching method: Application to multispectral chromosome image classification. In: 15th IEEE International Conference on Image Processing, ICIP 2008, October 12-15, pp. 1456-1459 (2008)

15. Cambell, N.: The decorrelation stretch transformation. Int. J. Remote Sensing 17, 19391949 (1996)

16. Amanatiadis, A., Kaburlasos, V.G., Gasteratos, A., Papadakis, S.E.: A comparative study of invariant descriptors for shape retrieval. In: IEEE International Workshop on Imaging Systems and Techniques, May 11-12, pp. 391-394 (2009) 